\title{
CAUSAS Y CONSECUENCIAS DE LA APATÍA EN UN GRUPO DE ESTUDIANTES DEL PROGRAMA DE PSICOLOGÍA FRENTE A LOS CURSOS DE MEDIACIÓN VIRTUAL
}

\author{
Miriam Yaneth Sánchez, Wilches ${ }^{71}$
}

\section{RESUMEN}

Conocer el porqué de la apatía frente a matricular cursos en mediación virtual que, periodo a periodo en época de pre matrícula, manifiestan los estudiantes de todos los programas y especialmente los de Psicología, se ha convertido para esta investigadora- que además cumple con funciones de tutora virtual-, en un reto en el sentido de revisar las causas que están generando este malestar que aunque se hacen evidentes, en muchos casos seguimos haciendo caso omiso a su afrontamiento.

Muchas investigaciones han estado orientadas a ir al fondo del problema, se han determinado causas y consecuencias y se han diseñado diferentes herramientas que le han permitido al estudiante y a los actores académicos utilizar nuevas estrategias pedagógicas y nuevos programas que mejoren la receptividad de todas las actividades diseñadas en un curso virtual.

El malestar que se manifiesta, también se trasmite de estudiantes antiguos a nuevos, se hace evidente en el no rotundo de algunos de ellos a matricular cursos virtuales y se cristalizó en la comunicación que a través de la red social Facebook registra: "No rotundo a obligar a la mediación virtual en la UNAD".

Todas estas inquietudes vienen generando consecuencias tanto negativas como positivas; se han revisado muchos de los procesos de aprendizaje y en especial el diseño de ambientes de aprendizajes autónomos y la construcción de escenarios digitales para la educación a distancia. En lo negativo, a partir de la situación generada, se busca contrarrestar la situación con un acercamiento pertinente y consecuente a las necesidades de los estudiantes y apoyar la práctica pedagógica que garantice acompañamiento académico y pedagógico en donde se hace evidente nuestra responsabilidad formativa.

Palabras clave: mediación, campus virtual, malestar, proceso de aprendizaje.

\begin{abstract}
Knowing the reason for the apathy that period to period in time of manifest pre registration students in all programs and especially those of psychology courses in mediation virtual register has been developed for this research also it expires with functions of virtual tutor,

\footnotetext{
71 Grupo de Investigación: Constructores de realidades humanas, Código del Grupo en Colciencias: COL0072139, Nombre del semillero: Línea de investigación: Factores asociados a la calidad de la Educación Abierta y a Distancia. Red de Investigación: Inteligencia colectiva y factores de calidad de la educación en ambientes digitales. UNAD.
} 
In a challenge in the sense of checking the reasons that are generating this discomfort that though they become evident in many cases we continue ignoring to his confrontation. Much research has been oriented in the sense of going to the root of the problem and has determined causes and consequences and are designed tools that have enabled the student and academic actors use new teaching strategies and new programs that improve the responsiveness of all activities designed in a virtual course.

This discomfort can also be transmitted from new and old students is evident in the resounding not enroll any students in virtual courses and communication through a social network Facebook records "resounding no to force the unad mediation in virtual "echoes some students.

All these worries come generating both negative and positive consequences, many of the learning processes have been checked and especially the design of environments of autonomous learning and the construction of digital scenes for the education distantly.

In the negative thing, from the generated situation, it is sought to offset by a pertinent and consistent approximation to the student's needs through the support of the pedagogic practice that guarantees academic and pedagogic accompaniment where our formative responsibility becomes evident.

Key words: mediation, virtual campus, discomfort, learning process.

\section{INTRODUCCIÓN}

En la Educación Superior a Distancia, la virtualidad se convierte en una de sus principales herramientas como alternativa para ofertar sus programas académicos y la articulación de las diferentes actividades en los proceso de aprendizaje de sus estudiantes.

Conocer las causas y consecuencias que generan apatía hacia los cursos académicos ofertados en mediación virtual, desde la percepción de malestar en periodo de pre matrícula en una gran mayoría de estudiantes, lleva a la investigadora a indagar el asunto en un grupo de estudiantes del programa de Psicología clientes potenciales y soporte de su usabilidad.

Este tema inquieta constantemente a la Universidad que cuenta con la mediación virtual, y se preocupa porque el producto ofertado cumpla con los estándares de calidad y de respuesta satisfactoria a las inquietudes manifiestas de sus usuarios, mejorando a través de su diseño curricular la optimización de la acción educativa, la comunicación, la aceptabilidad, la asequibilidad, en el Campus Virtual.

La indagación a través de la aplicación de un cuestionario nos permite conocer las causas y consecuencias de la apatía de este grupo de estudiantes que serán analizadas desde un proceso de intervención a la par que nos permita conocer si el grado de apatía disminuye o se mantiene. 


\section{OBJETIVO GENERAL}

Conocer las causas y consecuencias de la apatía que un grupo de estudiantes del programa de Psicología tiene con respecto a los cursos académicos en mediación virtual.

\section{OBJETIVOS ESPECÍFICOS}

Aplicar un cuestionario que permita obtener información con respecto a las causas que generan la apatía en cursos en mediación virtual.

\section{METODOLOGÍA}

Se aplica a esta investigación el tipo de estudio analítico descriptivo cuyo objetivo es el de analizar y describir situaciones reales insertas en el medio educativo de la Universidad.

Población: estudiantes del programa de Psicología.

Muestra: la muestra está representada por 50 estudiantes de diferentes periodos del programa de Psicología.

Fuentes de recolección de información: dentro de las Técnicas para recolectar información, se escoge la herramienta de entrevista estructurada estandarizada que aplica un cuestionario de diez preguntas estableciendo los criterios a analizar, teniendo en cuenta que el grupo escogido, cincuenta estudiantes, tienen los conocimientos adecuados, para responder en forma escrita y ser calificadas en una escala numérica según la gradación con el fin de conocer la intensidad del rasgo observado.

\section{RESULTADOS PARCIALES}

Pregunta 1. Su comentario con respecto a la mediación en Campus Virtual

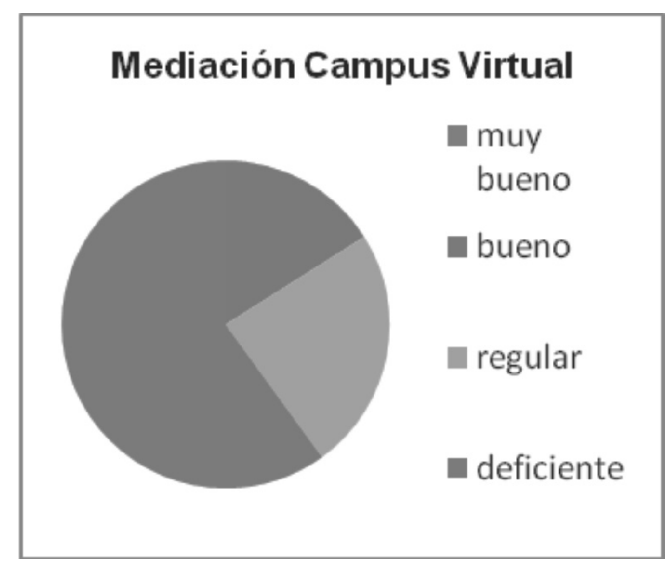


Un $60 \%$ de los estudiantes encuestados responde deficiente en el sentido de que ellos no tienen las habilidades para el manejo de estos cursos en plataforma y sienten que es imposición de la Universidad que los tienen que matricular, el $24 \%$ cree que es bueno pero no están completamente de acuerdo con esta mediación y un $16 \%$ están de acuerdo ya que sus conocimientos en herramientas telemáticas por el desempeño laborar y la disponibilidad de tiempo ayudan a que esta mediación resulte atractiva y de interés personal.

Pregunta 2. Su comentario con respecto a la conectividad al Campus Virtual

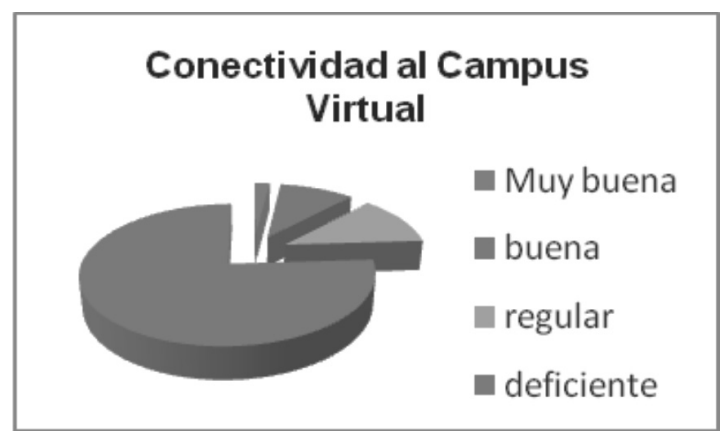

El 76\% de los encuestados tiene dificultades con la conectividad especialmente en fechas de cierre de actividades, sumado a esto el servicio regular que prestan las diferentes empresas de venta de internet, un $12 \%$ es regular por el tiempo que se toma para acceder al Campus Virtual la demora al conectar a los cursos virtuales, un 10\% manifiesta no haber tenido inconvenientes y el $2 \%$ restante acceder en horas de la madrugada donde no se presenta congestión.

Pregunta 3. Su comentario con respecto a la respuesta en la solución de problemas técnicos

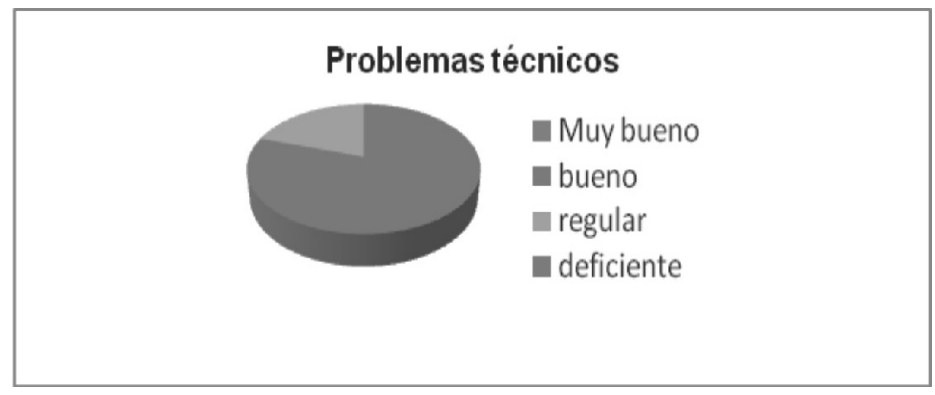

Un $80 \%$ comenta que hay una respuesta oportuna a la solución de problemas técnicos, pero para un $20 \%$ la respuesta no es oportuna y se llega a no obtener respuesta a tiempo. 
Pregunta 4. Su comentario con respecto a la capacitación en habilidades tecnológicas

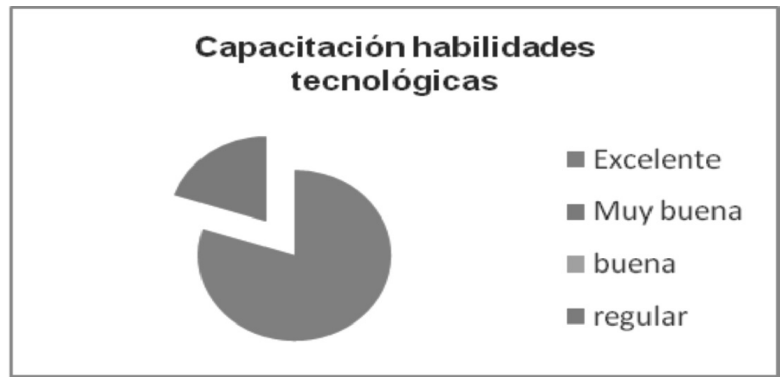

El $80 \%$ tiene un comentario excelente con respecto a que ha recibido capacitación oportuna y pertinente en el manejo tecnológico, igual el $20 \%$ que cree que ha sido muy buena.

Pregunta 5. Su comentario con respecto a la capacitación de tutor en soporte técnico

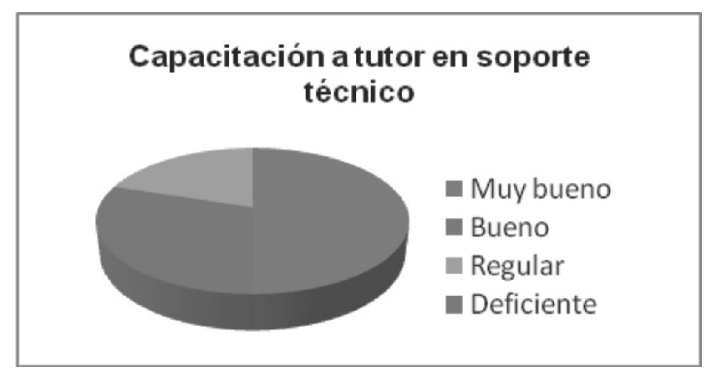

El 50\% tiene un comentario muy bueno con respecto a que los tutores si están capacitados en el soporte técnico del Campus virtual, el 30\% comenta que es buena la capacitación y un 20\% cree que es regular ya que los tutores no resuelven inquietudes en este sentido.

Pregunta 6. Su comentario con respecto al diseño de su Curso Virtual

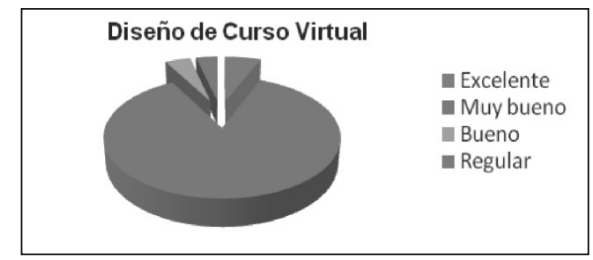

El 86\% hace un comentario muy bueno con respecto al diseño de su curso virtual, creen que tiene un buen impacto visual y es agradable entrar a él, otro $6 \%$ cree que su diseño es excelente en relación con otras páginas visitadas, un 4\% que es bueno, fácil de interpretar y, finalmente otro $4 \%$ que su diseño es regular y tiende a confundir al estudiante. 
Pregunta 7. Comentario con respecto al acompañamiento y asesoría por parte del tutor virtual en las diferentes actividades

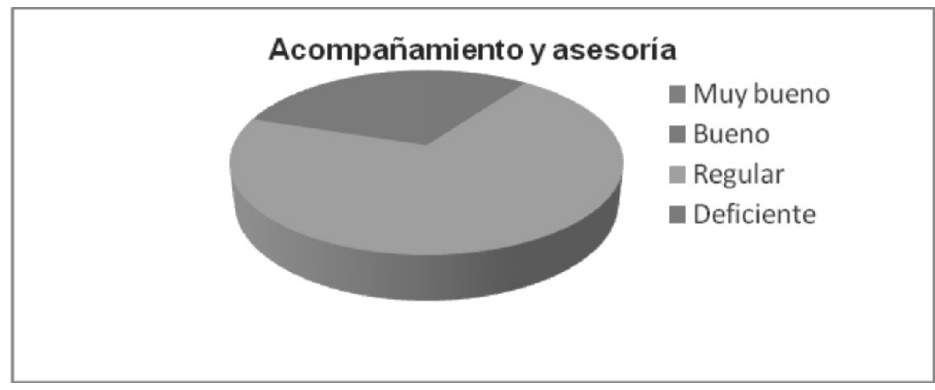

El 70\% refiere un comentario regular sobre la actividad de acompañamiento y asesoría virtual, los tutores no responden oportunamente las inquietudes especialmente en los Foros y mensajería interna creando molestia en los estudiantes que hacen sus esfuerzos por ingresar al Campus Virtual. Preocupa el 20\% sobre el comentario deficiente, porque se deduce que hay un grado de frustración en asumir los cursos virtuales y sólo un $10 \%$ siente que esta actividad ha sido comprometida por un grupo muy mínimo de tutores.

Pregunta 8. Su comentario con respecto al Trabajo Colaborativo

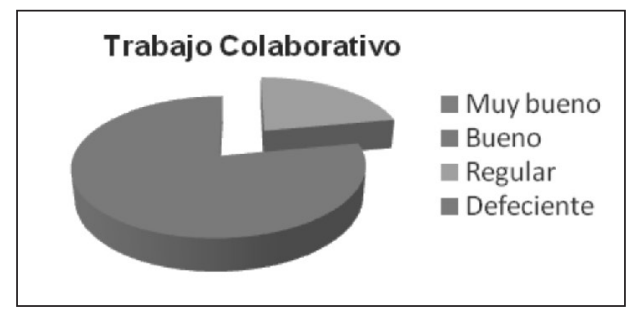

El 78\% de los estudiantes cree que la actividad de Trabajo Colaborativo es de las actividades más hartas ya que no se evidencia compromiso en enviar aportes, el tutor no revisa ni realimenta y se convierte esta actividad en la que más se demuestra apatía y corrobora el sentimiento de desgano de los estudiantes, el $22 \%$ restante comenta lo regular de esta actividad por la cantidad de actividades que se dejan para desarrollar en forma individual y en grupo, que a veces, resultan excesivas y que no reciben realimentación oportuna y adecuada. 
Pregunta 9. Su comentario con respecto a la calificación de actividades evaluativas y colaborativas

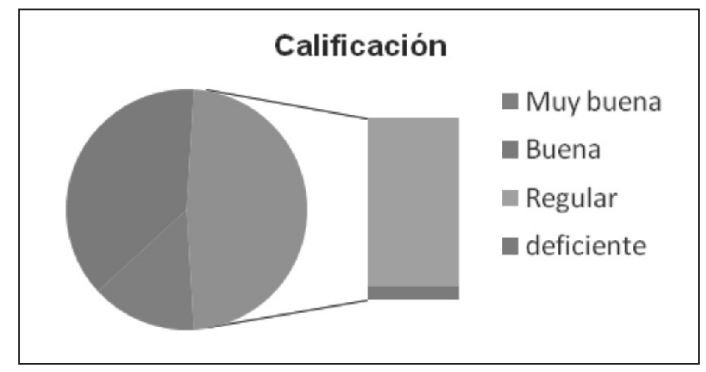

Un 48\%, casi la mitad de los estudiantes encuestados, comenta que las diferentes actividades evaluativas y de Trabajo Colaborativo, se califican muy a la ligera sin tenerse en cuenta, en mucho de los casos la rúbrica de evaluación, y los comentarios del tutor virtual no son pertinentes con lo que se está calificando. El 38\%, un porcentaje representativo, comenta que las actividades son bien calificadas aunque ratifican que la realimentación no es la mejor y un $14 \%$ que es muy buena, confirmando que se debe a su dedicación.

Pregunta 10. Comentario con respecto a la participación en el curso virtual

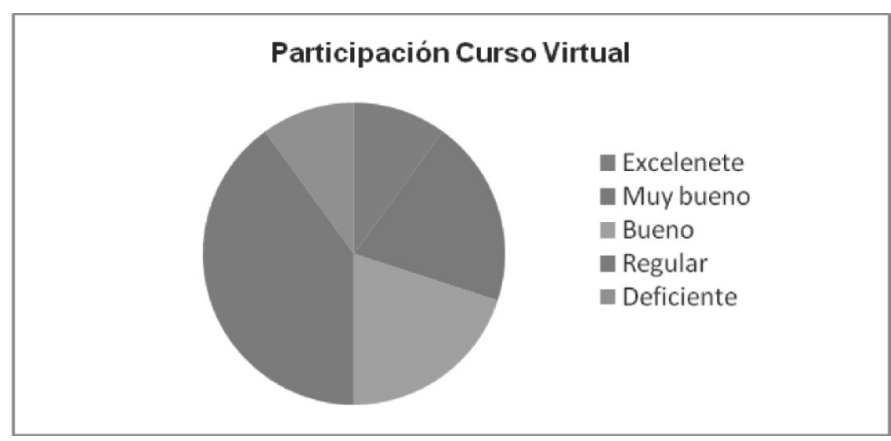

El comentario que hacen los encuestados con respecto a la participación en el Curso Virtual, arrojó los siguientes resultados: un $40 \%$ lo hace en forma regular, un $20 \%$ cree que su participación es buena, igualmente otro $20 \%$ que es muy buena y con $10 \%$ respetivamente en forma excelente y deficiente los demás.

\section{DISCUSIÓN}

Los comentarios de los estudiantes nos llevan a generar discusión en torno a:

- La frecuencia del $60 \%$, periodo a periodo siempre ha correspondido a las dificultades que presentan los estudiantes con respecto al manejo de las herramientas telemáticas, incluso evidenciando estudiantes que nunca han tenido acceso a usar un computador y 
menos a ingresar a la internet, esto implica una limitación y por ende se debe considerar como una causa de la apatía hacía los cursos en mediación virtual; debemos comentar, además, que el curso de entrenamiento está obligando al estudiante a sacar los 400 puntos mínimos requeridos pero son, a veces otros compañeros, quienes presentan esta prueba o se hace en serie bajo la orientación de otro estudiante, no aplicando la rigurosidad académica de este ejercicio, se realiza " por que toca cumplir".

- El 88\% del estudiantado sigue apuntando a las dificultades que a diario se presentan con la conectividad a la plataforma tecnológica aún con los avisos que se fijan en el Campus Virtual de la efectiva conectividad. Los estudiantes siguen mostrando mucho desinterés con respecto a tantos problemas técnicos que deben sortear día a día para poder ingresar, evidenciando motivos suficientes de su desgano y desdén para cumplir con las actividades programadas. Causa curiosidad el $2 \%$ de estudiantes, debo incluir algunos tutores compañeros que hemos accedido en horas de la madrugada a cursos virtuales, porque es el único momento en el que se puede acceder sin muchos inconvenientes.

- Los frecuentes problemas técnicos en un $80 \%$ de las respuestas no se atienden en forma oportuna, siendo consecuente con el punto anterior que no hay una respuesta oportuna a la gran mayoría de casos reportados.

- Casi el 100\% del comentario con respecto a la capacitación en habilidades tecnológicas es favorable, aquí se destaca el compromiso del personal académico en acompañar y orientar oportunamente a los estudiantes en el acceso al Campus Virtual y más concretamente en lo referente a los cursos en mediación virtual. Los estudiantes manifiestan su complacencia con la Sala de Sistemas que está adecuada con los equipos necesarios, la flexibilidad de los horarios y la disposición del personal que la atiende. Para fortalecer esta actividad se ha asignado un horario por día a cada tutor para atender a los estudiantes que acuden a sala de sistemas y a otros se les ha asignado la responsabilidad de un curso virtual para que esté atento en orientar el fin de semana.

- Pensamos que frente al 20\% del comentario desfavorable de que no hay una buena capacitación técnica de los tutores, el $80 \%$ ratifica que se evidencia una muy buena formación, con algún malestar frente a no obtener respuesta oportuna a sus inquietudes.

- Un 96\% hace comentario favorable con respecto al diseño de los cursos, un porcentaje muy bajo del $4 \%$ cree que algunas actividades tienden a confundir, además de que no se actualizan fechas y presentan errores de redacción y ortografía.

- Preocupa que un comentario del $90 \%$ con respecto a la atención de tutoría virtual, no sea tan favorable, en este periodo las principales reclamaciones que han presentado los estudiantes están referidas a la no respuesta oportuna a sus inquietudes, igual que el 
malestar con la revisión y realimentación de aportes no se realiza de forma oportuna; un porcentaje muy mínimo resalta la labor que realizan los tutores.

- La actividad de Trabajo Colaborativo merece un comentario muy especial ya que es la actividad crítica para los estudiantes, se sienten defraudados al tener que participar en una actividad donde no se aporta con la respectiva responsabilidad, algunos aportes no cumplen con los parámetros exigidos, muchas actividades para desarrollar en tan poco tiempo que no permiten una apropiación adecuada, la ampliación de fechas que afecta los aportes oportunos de envió, la no respectiva revisión y realimentación por parte de los tutores hacen que un comentario del $80 \%$ ratifique el malestar que esta actividad causa en la mayoría de estudiantes, sumado al $20 \%$ restante que creen que es una actividad que se atiende muy regularmente y que debe de ser reevaluada.

- Un $48 \%$ casi la mitad de los estudiantes encuestados comenta que las diferentes actividades evaluativas y de Trabajo Colaborativo, se califican muy a la ligera sin tenerse en cuenta, en mucho de los casos, la rúbrica de evaluación y que los comentarios del tutor virtual no son pertinentes con lo que se está calificando, el $38 \%$ un porcentaje representativo, comenta que las actividades son bien calificadas aunque reafirman que la realimentación no es la mejor y un $14 \%$ que es muy buena ratificando que se debe a su dedicación reconocida por el tutor.

- Las actividades evaluativas presentan inconsistencias en la formulación de las preguntas y en algunos casos las opciones de respuesta no corresponden, lo que deja al estudiante a la expectativa si su respuesta ha sido respondida de forma correcta.

- Llegamos a otro punto crítico como es el de la participación de los estudiantes en los cursos virtuales. Promediando, un $60 \%$ comenta que lo hace de forma muy regular lo que denota que el estudiante participa, acosado, en las fechas próximas al cierre de las actividades. Se presenta una dualidad en un $10 \%$ excelente y un $10 \%$ deficiente, el comentario respectivamente obedece a quienes pueden ingresar a la plataforma casi que a diario y quienes sólo participan cada vez que se pueden conectar o vienen al CEAD, el no contar con computador, no tener acceso a la internet entre algunos factores incidentes.

\section{PROPUESTA DE INTERVENCIÓN}

\section{Fase 1. Recolección de información}

Instrumento a aplicar: Encuesta estructurada cerrada

Población objeto: 50 estudiantes del programa de Psicología

Sistematización y tabulación de resultados

Diagnóstico 


\section{Fase 2. Intervención}

Curso virtual matriculado por los estudiantes

Acompañamiento dos veces por semana a curso virtual a 20 estudiantes

Acompañamiento cada 8 días a curso virtual a 10 estudiantes

Acompañamiento cada 15 días a curso virtual a 10 estudiantes

Acompañamiento $1 \mathrm{vez}$ al mes a curso virtual a 5 estudiantes

No acompañamiento en curso virtual a 5 estudiantes.

\section{Fase 3. Evaluación}

Interpretación y análisis de resultados

Conclusiones

Esta información nos lleva a proponer un plan de intervención basado principalmente en hacer un acompañamiento a los estudiantes según lo manifestado y que permita mejorar su motivación con respecto a su participación. Hay un tiempo de 4 semanas y se distribuyen los estudiantes, así:

Tabla 1. La evaluación del acompañamiento en el Campus Virtual

\begin{tabular}{|c|c|}
\hline Número estudiantes & Días de acompañamiento \\
\hline 20 & 8 \\
\hline 10 & 4 \\
\hline 10 & 2 \\
\hline 5 & 1 \\
\hline 5 & Ningún día \\
\hline
\end{tabular}

Con respecto al desarrollo del proceso de acompañamiento evaluamos los nuevos comentarios de los estudiantes acerca del acceso al Campus Virtual, realizando el respectivo análisis, principalmente con la usabilidad de la plataforma y los contenidos on-line como atractivo de esta intervención a partir de la mediación comunicacional real.

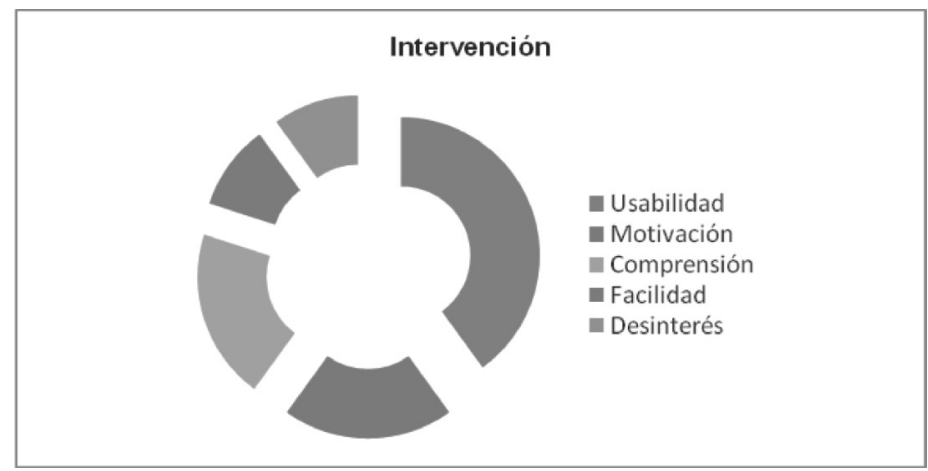

Gráfica 1. Resultados de la intervención 
Los resultados de la intervención nos permiten establecer el siguiente análisis: un 40\% adquirió una mejor usabilidad del Campus Virtual, un 20\% mejoró el grado de motivación, otro $20 \%$ la comprensión de cada una de las actividades y su desarrollo, un $10 \%$ cree que es fácil acceder a la plataforma y finalmente otro $10 \%$ sigue manteniendo un desinterés por causas externas ya que en el lugar de residencia no hay conectividad a internet y sólo lo realizan cada vez que vienen al CEAD. Aplica, para este ejercicio, la evaluación cualitativa ya que tiene como finalidad una comprensión holística de la realidad: hacer una lectura general de los comportamientos, actitudes, habilidades y competencias adquiridas, los resultados de la evaluación se expresan a través de juicios de valor en los que se hace interpretación de los saberes aprendidos por los estudiantes y variables contextualizadas más amplias y complejas de la acción educativa ejercida por el tutor acompañante.

\section{IMPACTO DEL TRABAJO EN EL CONTEXTO UNADISTA}

Indagar sobre las causas y consecuencias que vienen generando malestar en muchos estudiantes frente al acceso al Campus Virtual permite, desde la recolección de información, abrir un canal efectivo de comunicación donde las partes involucradas afronten y resuelvan unas causas evidentes que permitan grados de satisfacción y por ende desde la institución acciones de mejora en hallazgos que son repetitivos de reclamaciones y que deben de evaluarse y dentro de los planes operativos tenerse en cuenta como acciones que mejoren el quehacer académico y se permita una retención de estudiantes motivados frente a su proceso formativo.

\section{CONCLUSIONES}

Después de hacer el anterior análisis se recomienda hacer una revisión minuciosa de los Módulos como referente teórico y su aplicabilidad.

Además, diseñar y aplicar una encuesta a todos los estudiantes que han matriculado cursos en mediación virtual como ejercicio que permita medir el grado de motivación, tomar decisiones para deconstruir algunos aprendizajes y dar respuesta oportuna y satisfactoria a las distintas reclamaciones en este sentido.

Por otro lado, adelantar un ejercicio de acompañamiento práctico en tiempo real y con el aval de cada estudiante que facilite su tiempo e interés.

De otra parte, evaluar desde la práctica la intervención de tutores asignados en Campus virtual en el cumplimiento de su labor sustantiva.

Asimismo, los problemas recurrentes con la plataforma deben de ser revisados continuamente ya que es allí en donde se genera el mayor grado de inconformidad.

Se debe devolver la motivación a más de la mitad de los estudiantes frente al manejo de la plataforma tecnológica. 
Es necesario revisar los diferentes roles de los tutores con la finalidad que se asigne la actividad virtual en forma pertinente y consecuente.

Se hace imperativo reconocer a aquellos tutores que se destacan en la asesoría, acompañamiento y seguimiento a cada estudiante en Campus Virtual.

Revisar la carga académica de los tutores que se les permita que su tiempo contratado sea únicamente para atender adecuadamente y con calidad los cursos virtuales asignados.

El hecho de no disponer de conectividad las 24 horas del día y contar con un acceso regular a la web, no permite cumplir con las actividades adecuadamente y en las fechas establecidas lo que seguirá generando malestar en los otros estudiantes que se permiten ser cumplidos con el envió de sus aportes pero por razones obvias de otros estudiantes terminan entrando el día que puedan atrasando el proceso formativo de unos y siendo mediocres con el proceso de los otros.

Finalmente, que la información recolectada sirva como elemento EVALUADOR y este permita planificar y redireccionar muchas de las actividades pedagógicas en la planeación y toma de decisiones.

\section{REFERENCIAS BIBLIOGRÁFICAS}

BARRAGÁN, Rossana y otros. (2001), Formulación de proyectos de investigación. Serie PIEB formación. 2da. Edición. Bolivia.

CERDA, Hugo. (2002), Los elementos de la investigación. Como reconocerlos, diseñarlos y construirlos. Editorial EL BUHO LTDA. Bogotá.

VELA GONZÁLEZ, Pedro Antonio (2009), Módulo de Evaluación en Entornos virtuales.

RESTREPO GÓMEZ, Bernardo. Una variante pedagógica de la investigación acción-educativa.

http://www.aces-andalucia.org/doc/cabero.doc

http://tecnologiaedu.us.es/nweb/htm/cuerpo.htm 